\title{
A CMOS transmitter front-end with digital power control for WiMAX 802.16e applications
}

\author{
Yu-Hsuan Liu, Hsiao-Chin Chen, Tao Wang and Shey-Shi Lu \\ Graduate Institute of Electronics Engineering, National Taiwan University
}

\begin{abstract}
A $5.8 \mathrm{GHz}$ transmitter front-end comprising a quadrature modulator, a variable gain amplifier and an on-chip output balun in CMOS 0.18-um technology is presented. The quadrature modulator adopts cross-coupled type micro-mixer, and the measured $3^{\text {rd }}$-oder rejection is $32 \mathrm{~dB}$ under input voltage swing of $250 \mathrm{mVpp}$. With four-bit control words, a 16-step linear-in-dB output power is realized to achieve a dynamic range of $27 \mathrm{~dB}$. A single-ended type output is accomplished by an on-chip 3:1 transformer and the output matching network is therefore simplified.
\end{abstract}

Key Words: RF, transmitter, VGA, digital control, linear-in-dB

\section{INTRODUCTION}

Recently, the IEEE has come up with a new standard, 802.16e, also known as WiMAX, which is a short name for World Interoperability for Micro-wave Access. WiMAX is a broadband wireless technology which promises not only high data-rates but also long connection distances. Standards and applications based on this attractive technology are scheduled for initial deployment in mid-to-late 2005. Since WiMAX adopts OFDM modulation scheme for high data-rates, the modulated signals will display a high Peak-to-Average Power Ratio (PAPR) and a strict linearity requirement in transmitter can be expected. Besides, 802.16e support a roam mode which provides each user a connection with cell phone quality, therefore, a wide output power range is necessary. In this paper, the design issues such as the linearity, the output power range, and the linear-in- $\mathrm{dB}$ gain control are considered and discussed. To facilitate the integration with off-chip power amplifiers in general purposes, an on-chip transformer of 3:1 turn-ratio is used to convert the differential output signal of the variable gain amplifier (VGA) to a single-ended signal.

\section{CIRCUIT BLOCK}

The architecture of the transmitter front-end is shown in Fig. 1. A quadrature modulator is followed by a VGA which contains an on-chip transformer of 3:1 turn-ratio. The base-band signal is up-converted by the modulator and then amplified by the two-stage VGA. Four digital bits are manipulated to control the gain of the VGA in a linear-in-dB fashion. The LO signal is applied externally through a rat-race hybrid, and converted into quadrature signals by a poly-phase filter. 


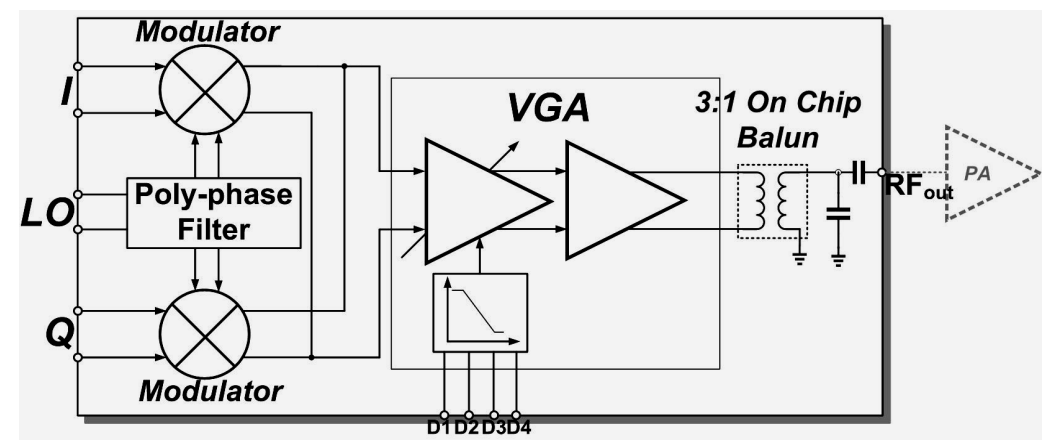

Fig. 1 Transmitter Front-end Architecture

The schematic of the modulator is shown in Fig. 2. The modulator is based on the asymmetry class-AB topology of the MicroMixer [1] and the transconductance stage is cross-coupled to maintain the symmetry in base-band signals. With the class- $\mathrm{AB}$ structure, the modulator can handle larger base-band signals and provide a wider input dynamic range. With proper device selection, the nonlinear phenomenon respectively occurs in the common-gate and the common-source MOSFETs can be cancelled by each other under a particular bias condition [1].

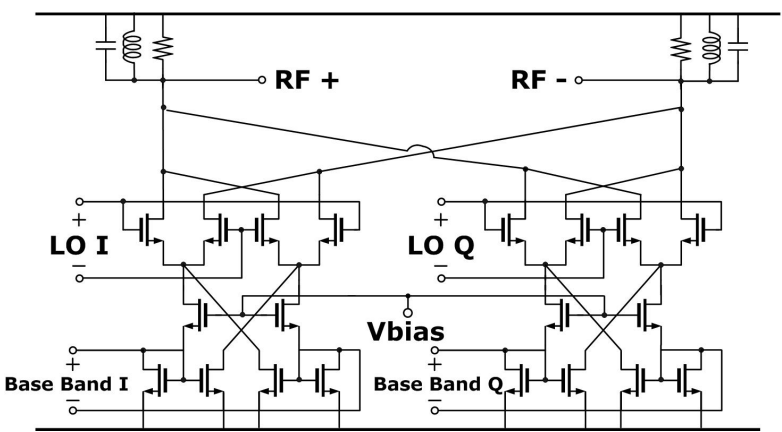

Fig. 2 Modulator schematic

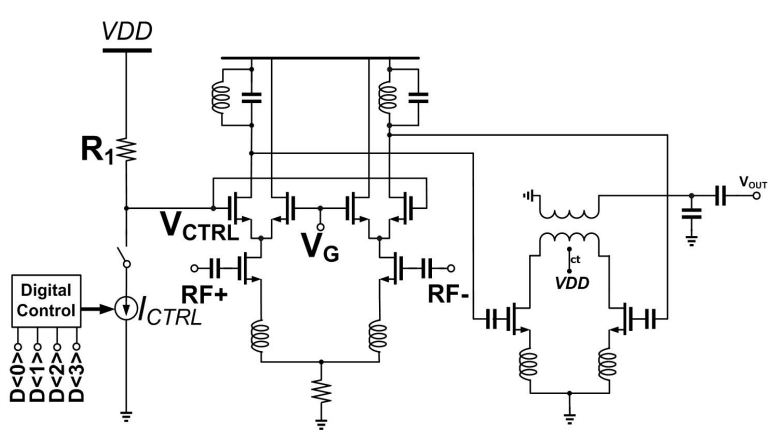

Fig. 3 VGA schematic.

The gain control is realized with a current steering structure in the first stage of the VGA as shown in Fig.3. The on-chip transformer offers its primary coil as the load inductors of VGA's second stage, thus accomplishes a differential-to-single-ended conversion. The transformer was simulated by momentum with its physical layout shown in Fig.4 [2].

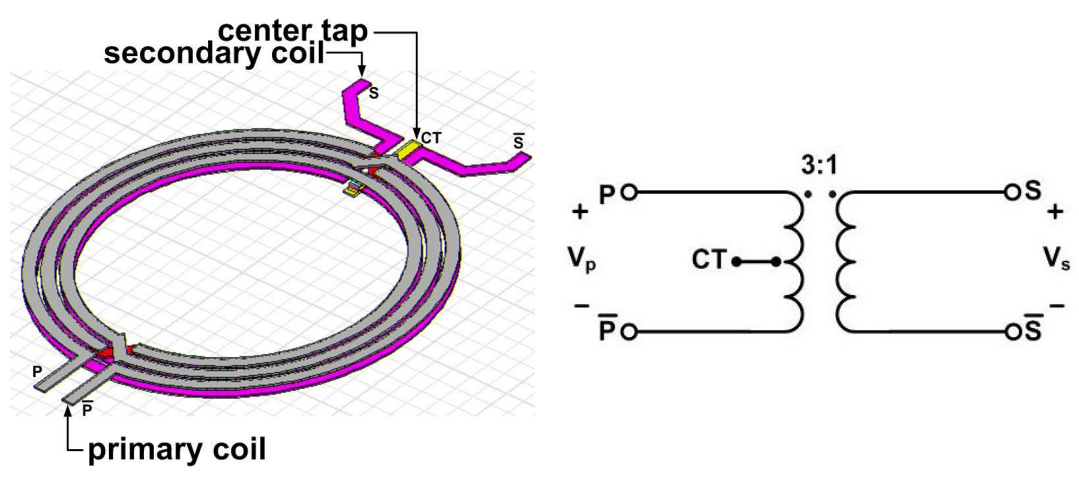

Fig. 4 Physical layout and schematic symbol of the transformer

The supply voltage can be applied via the center tap of the primary coil. A turn-ratio of 3:1 is selected 
to simplify the impedance matching between the output stage and the 50-ohm.

16 gain steps are achieved by the digital-control block shown in Fig. 5. The decoder first translates the four digital bits into 18 control words fed to the digital-to-analog-converter (DAC), which is composed of seven binary weighted current sources with source-coupled-logic switches. The DAC can create an output current by which the control voltage $V_{\text {CTRL }}$ is adjusted. Degeneration resistors are used to reduce the mismatches between the current sources and an irregular coding is also utilized to minimize the gain step error.

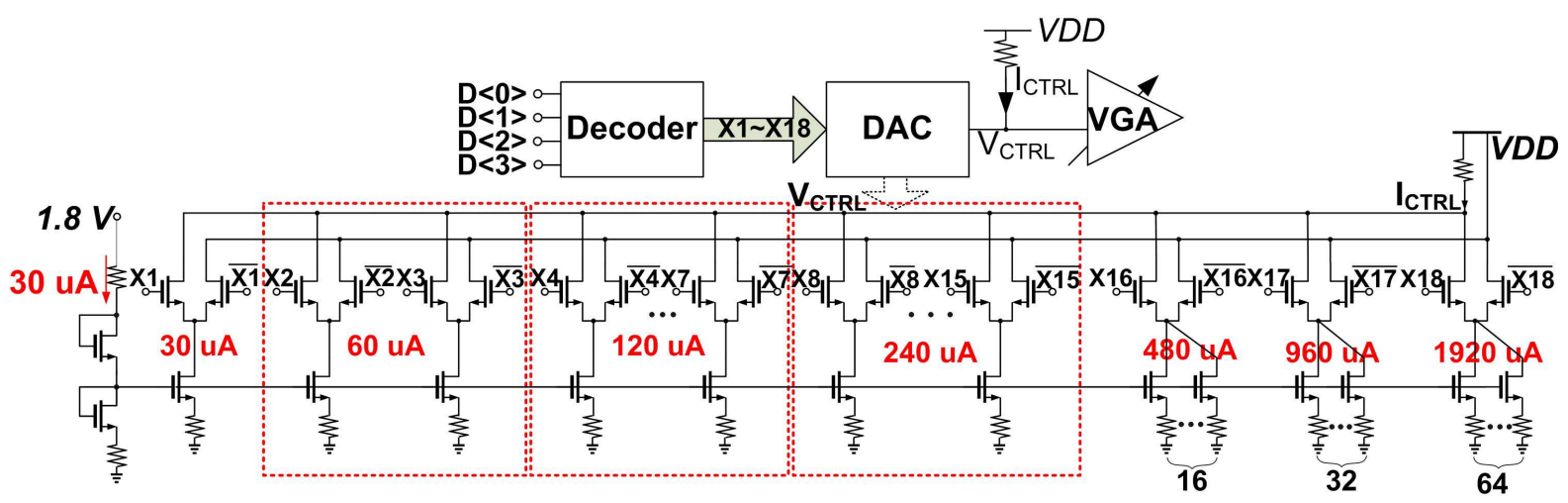

Fig. 5 The digital control block and the schematic of DAC.

\section{Simulation AND MEASUREMENT RESUltS}

The transmitter front-end draws $53.3 \mathrm{~mA}$ from a $2.8 \mathrm{~V}$ supply. The quadrature base-band signals are applied by R\&S AMIQ signal generator. The measured output spectrum of the modulator is shown in Fig. 6. The $\mathrm{LO}$ rejection is $28.0 \mathrm{~dB}$, the sideband rejection is $13.7 \mathrm{~dB}$, and the third order rejection is $31.9 \mathrm{~dB}$. The OIP3 of the VGA is about $15 \mathrm{dBm}$ as shown in Fig. 7.

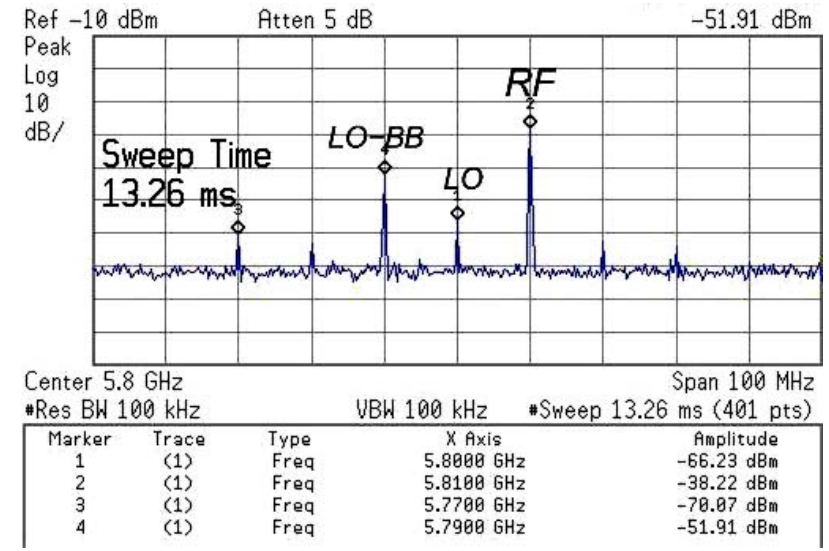

Fig. 6 Measured output spectrum of the modulator.

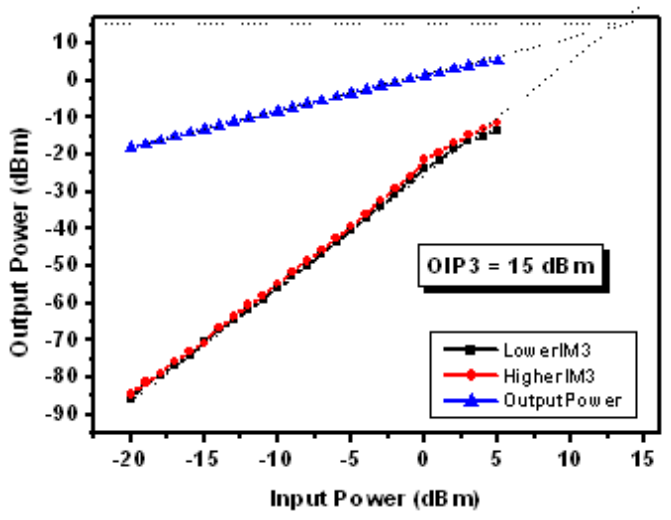

Fig. 7 OIP3 measurement result.

Fig. 8 shows the simulated dB-linear gain at each step. The linear-in-dB gain control characteristic is achieved with a $2.13-\mathrm{dB}$ step and a $\pm 0.8 \mathrm{~dB}$ gain error. The digital controllable range is over $33 \mathrm{~dB}$. The measured dynamic range is about $27 \mathrm{~dB}$ as shown in Fig. 8. Although the gain range is smaller than the simulation result, it is wide enough for general applications. 


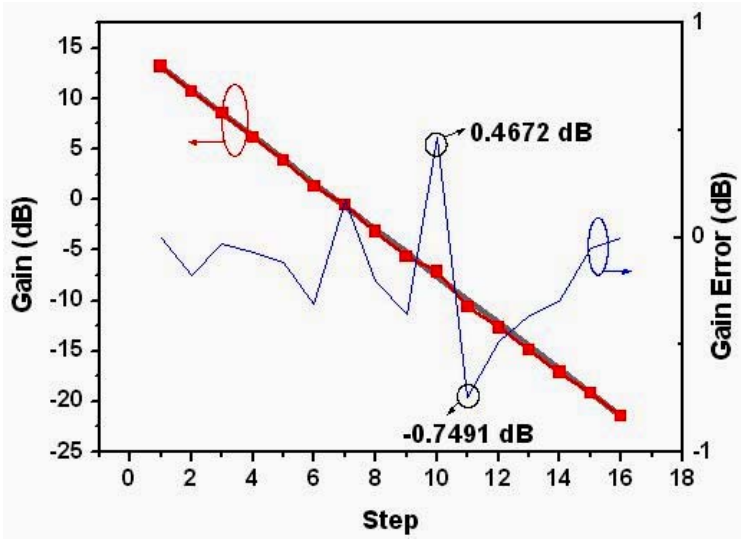

Fig. 8 Simulated Gain versus input control words.

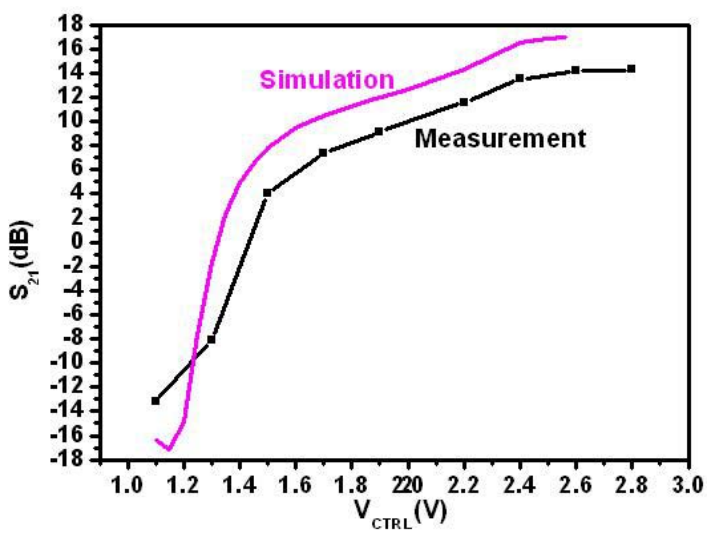

Fig. 9 Dynamic range of VGA.

The die photograph and evaluation board of the transmitter front-end are shown in Fig. 9 and Fig. 10, respectively. The total die area is $2.4 \mathrm{~mm} \times 1.1 \mathrm{~mm}$.

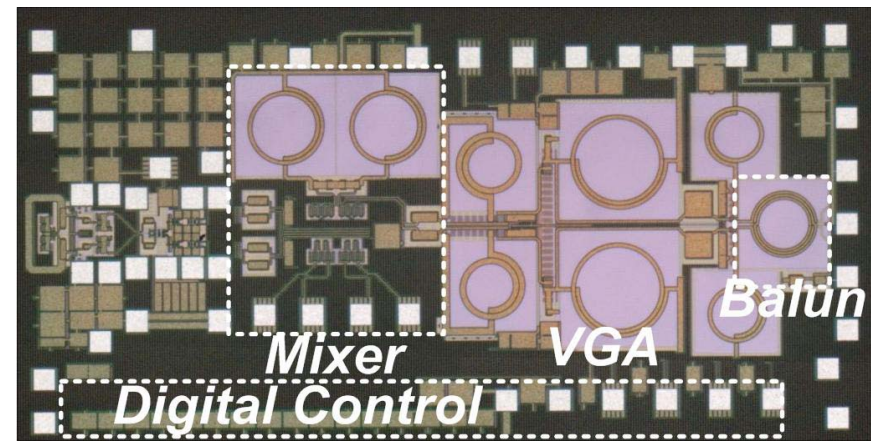

Fig. 10 Die photograph of the transmitter front-end.

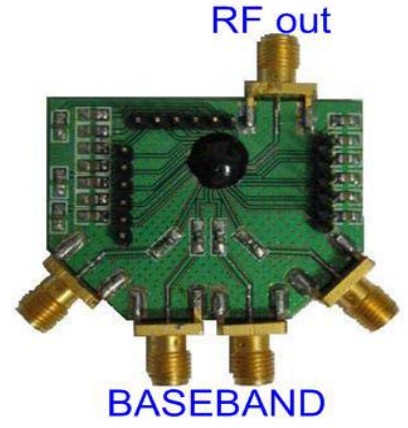

Fig. 11 Evaluation board.

\section{Conclusions}

A 5.8GHz CMOS transmitter front-end with digital linear-in-dB gain control is presented in this paper. The output signal demonstrates good performances in unwanted spur rejection and an OIP3 of about $15 \mathrm{dBm}$. This transmitter also has an output dynamic range of $27 \mathrm{~dB}$.

\section{ACKNOWLEDGMENT}

Financial support from 91EC17A05-S10017, NSC 94-2215-E-002-032 and NSC 94-2218-E-002-045 is appreciated. The authors would like to thank CIC for chip fabrication and NDL for chip measurement.

\section{REFERENCES}

[1] Barrie Gilbert, "The MICROMIXER: A Highly Linear Variant of the Gilbert Mixer Using a Bisymmetric Class-AB Input Stage," IEEE J. SOLID-STATE CIRCUITS, vol. 32, no. 9, Sept. 1997

[2] Alessandro Italia, Egidio Ragonese, Luca La Paglia, and Giuseppe Palmisano, "A 5-GHz High-Linear SiGe HBT Up-converter with On-chip Output Balun," IEEE Radio Frequency Integrated Circuits Symposium, pp. 543-546, 2004. 